\title{
FAST MEASUREMENT OF SEP FOR MONITORING SPINAL CORD DURING SCOLIOSIS
}

\author{
${ }^{1}$ K.S.M. Fung, ${ }^{1}$ M.H. Du, ${ }^{1}$ W.Q. Liu, ${ }^{1}$ F. H.Y. Chan, ${ }^{1}$ F.K. Lam, ${ }^{2}$ D.K. Luk and ${ }^{2}$ Y. Hu \\ ${ }^{1}$ Department of Electrical and Electronic Engineering, The University of Hong Kong, Hong Kong \\ ${ }^{2}$ Department of Orthopaedic Surgery, The University of Hong Kong, Hong Kong \\ E-mail: fhychan@eee.hku.hk
}

\begin{abstract}
Recently there has been considerable interest in the use of somatosensory evoked potential (SEP) for monitoring the functional integrity of the spinal cord during surgery such as scoliosis. This paper describes a monitoring system and signal processing algorithms, which consist of an artificial neural network filter and a wavelet signal enhancer developed to enhance the signalto-noise ratio (SNR) of surface recorded SEP. Our system allows fast detection of change in SEP's peak latency, amplitude and signal waveform, which are the main parameters of interest during intra-operative procedures.
\end{abstract}

Index Terms - scoliosis, SEP monitoring, line interference canceling, wavelet analysis.

\section{INTRODUCTION}

Nowadays, spine operation becomes a common surgery. Scoliosis is a major surgery to correct spinal deformity, which carries a significant risk of permanent neurological damage due to lesions of the spinal cord. Spinal cord is an integral part of central nerves system embedded inside the spine. It is the only pathway to transmit neurological signals from peripheral nerve to cerebral cortex. Injury of the spinal cord is irreversible. In a recent survey on spinal cord monitoring, the rate of neurologic injury has been increased to $1.6 \%[1]$. It should be noted that the cost of long-term care for a quadriplegic has been estimated at over US\$150,000 annually. Therefore, a huge sum of money is spent in the caring of patients with spinal cord lesion. The survey also reported that $72 \%$ of these cases could be foreseen in interoperative monitoring. In order to avoid the lesion, it is important to employ a system to monitor the status of spinal cord during such operations.

Intraoperative monitoring of somatosensory evoked potentials (SEP) is a significant technique that has shown considerable promise and it can greatly improve the margin of safety during surgical procedures around the spine[2]. In order to minimize disturbance to doctors and reveal the most recent status of spinal cord, SEP is monitored between surgical procedures. The permanent damage to the nervous system could be avoided if the change of SEP could be detected. Despite the powerful capabilities of the technique, it suffers from a major drawback in that the SNR of the observed response is very poor. Usually, the signal recorded with surface electrodes has its SNR less than unity. The major sources of interference come from background activities of the nerves system and electrical interference from peripheral equipment attached to the patient. In this paper, we described the system and algorithms developed to measure SEP in real time.

\section{THE METHOD}

\section{A. Monitoring System}

Conventional EP machine can elicit SEP response by electrically stimulating a peripheral nerve and recording it over the cerebral cortex or spinal cord as the stimulus travels towards the central nervous system. The built-in ensemble averaging (EA) circuit suppresses the undesired noise, which is independent to stimulus. Usually 100 trials should be averaged to measure the SEP peak latency and amplitude. Thus, real-time monitoring of SEP is impossible and the measurement of SEP may disturb the operation processes. Pre-filtering of the recorded signal is therefore necessary to shorten the measurement time.

In order to overcome the shortcomings of conventional EP machine, we developed a SEP recording and monitoring system. The system is composed of a pre-amplifier, an A/D converter and a microcomputer. The pre-amplification is accomplished by the pre-amplifier of Dantec Cantata EP machine. Its main module can control the bandwidth and gain of the signal. The A/D card and the microcomputer used are DT2831 and Pentium PC respectively. SEP is digitized by the $\mathrm{A} / \mathrm{D}$ converter and then transmitted to the microcomputer for digital signal processing. SEP measurement is achieved by artificial neural network filtering of line interference and wavelet analysis. EA should be applied when further SNR enhancement is necessary.

\section{B. Line Interference Canceling}

By inspecting the recorded SEP, we can observe that the signal is heavily interfered by a $50 \mathrm{~Hz}$ sinusoidal component. Inside the operation theatre, many electrical instruments and accessories are attached to the patient for monitoring the electrocardiogram, breath, temperature, and blood oxygen saturation, etc. Causes of such power-line 
interference include magnetic induction, displacement currents in leads or in the body of the patient and equipment interconnections and imperfections. The conventional method of eliminating line interference is through the use of a fixed center notch filter characterized by a unit gain at all frequencies except at the line frequency, where its gain is zero [4]. Unfortunately, only the average line frequency over a period, say 24 hours, is fixed. The instantaneous frequency may vary from time to time. With the line interference available, Widrow et. al. proposed a solution using the interference as reference to adjust an adaptive filter [5].

Using the concept of adaptive noise canceling, an alternative filtering algorithm employing artificial neural network (ANN) is designed [6]. The ANN is trained to produce a replica of line interference. Thus, recording of power line signal is unnecessary. Filter output is produced by subtracting the estimated interference from the observed signal. The output, which is also called error signal, is fed back to the network for on-line training. Fig. 1 shows the application of an ANN in canceling sinusoidal interference. The ANN has three layers. Input of the network is time instant, $k$. The hidden layer is composed of a pair of sinusoidally activated hidden nodes with their phase separated by $\pi / 2$. Output of hidden nodes at time instant $k$ is

$$
\mathbf{H}=\left[\sin \left(2 \pi f_{i} \frac{k}{f_{s}}\right) \cos \left(2 \pi f_{i} \frac{k}{f_{s}}\right)\right]^{T}
$$

where $f_{s}$ and $f_{i}$ are sampling frequency and internal frequency of hidden nodes respectively. $f_{i}$ could be $50 \mathrm{~Hz}$ or $60 \mathrm{~Hz}$ depending on different countries. $\mathbf{H}$ is linearly combined with connection weights $\mathbf{W}=\left[\begin{array}{ll}w_{s} & w_{c}\end{array}\right]^{T}$ at output layer to produce an estimate of interference signal:

$$
y=\mathbf{H}^{T} \mathbf{W}
$$

Define an error signal to be the difference between observed signal and ANN output,

$$
e=d-y
$$

The network is trained on-line to minimize the squared error using gradient descent method with respect to weight vector. Therefore update of weight vector is given by

$$
\Delta \mathbf{W}=-\mu \frac{\partial e^{2}}{\partial \mathbf{W}}=2 \mu e \mathbf{H}
$$

$\mu$ is the convergence rate determining the speed and stability of the learning process. Analysis in [5] showed that the criteria for $\mu$ to ensure the convergence of training is

$$
0<\mu<\frac{1}{3 \operatorname{tr}\left[\mathbf{H H}^{T}\right]}=\frac{1}{3}
$$

By expanding and rearranging Eq. (2) we obtain

$$
y(k)=A \sin \left(2 \pi f_{i} \frac{k}{f_{s}}+\phi\right)
$$

where $A=\sqrt{w_{s}^{2}+w_{c}^{2}}$ and $\phi=\tan ^{-1}\left(w_{s} / w_{c}\right)$. Note that the training allows the weights to adjust in the way reproducing the power line interference with varying amplitude and phase for cancellation.

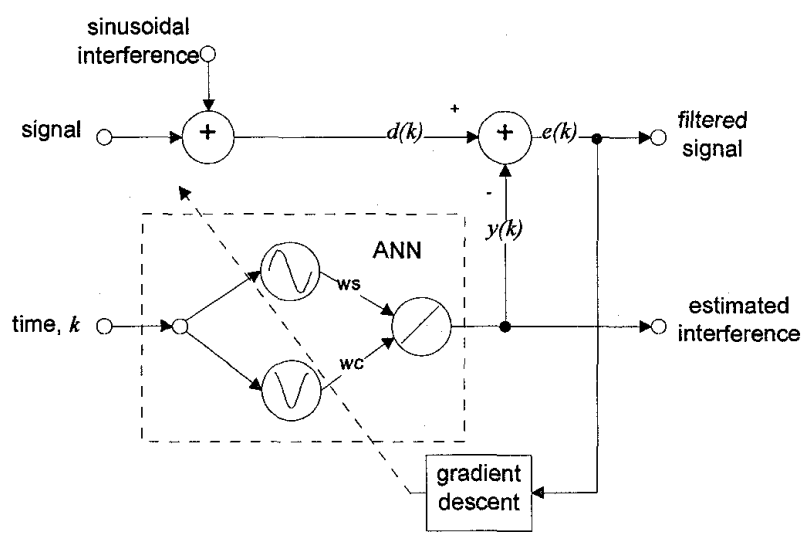

Fig. 1. Schematic diagram of the artificial neural network filter.

\section{Estimation of SEP from Background Nervous Activities}

Apart from the power line interference, the background on-going activities of the nerves system are the other disturbances that hamper SEP estimation. The estimation of SEP waveform buried in noise has received considerable attention in the literature. Among them, monitoring of SEP latency is realized by wavelet analysis [7]. In fact, wavelet analysis could be considered as the correlation between wavelet base of different scale and the SEP signal. It can reflect change in SEP from normal reference that can be recorded a priori via preoperative measurements. Based on the SEP, an appropriate wavelet base is formulated. It is used as the reference for detecting changes. During the operation, stimuli are applied to the patient. Any change in SEP could be monitored in the wavelet analysis of the recorded signal. In this paper, the wavelet representation proposed by Daubechies [8] is employed. Let $\phi(x)$ be a scale function and $\psi(x)$ be the corresponding wavelet. Defining the coefficients of filters $\mathrm{H}$ and $\mathrm{G}$ as follows,

$$
\left\{\begin{array}{l}
h(n)=2^{-1 / 2} \int d x \phi\left(\frac{1}{2} x\right) \phi(x-n) \\
g(n)=2^{-1 / 2} \int d x \psi\left(\frac{1}{2} x\right) \psi(x-n)
\end{array}\right.
$$

Suppose the data points of the $i$-th SEP trial is $\left\{x_{i}(0), x_{i}(1) \cdots x_{i}(N-1)\right\}$. For clarity, let it be $\left\{c_{0}^{0}, c_{1}^{0} \cdots, c_{N-1}^{0}\right\}$, so we have the decomposition formula: 


$$
\left\{\begin{array}{l}
c_{k}^{j}=\sum_{n} h(n-2 k) c_{n}^{j-1} \\
d_{k}^{j}=\sum_{n} g(n-2 k) c_{n}^{j-1}
\end{array}\right.
$$

where $j$ indicates the scale, $\left\{c_{k}^{j}\right\}$ is the coarse signal, and $\left\{d_{k}^{j}\right\}$ is detailed signal. The corresponding reconstruction formula is:

$$
c_{n}^{j-1}=\sum_{k} h(n-2 k) c_{k}^{j}+\sum_{k} g(n-2 k) d_{k}^{j}
$$

In real time monitoring, SEP's latency shifting and its amplitude changing are the most important. We should choose as few as possible filter coefficients for fast computation and the waveform of the chosen wavelet should have similar peaks and troughs. We found that the peaks' distribution in the inverted 4 coefficients Daubechies' wavelet is the most similar to those of averaged SEP signal around the latency area (Fig. 6). Obviously, with the wavelet base suitably selected, the SEP could be reconstructed with less number of trials.

\section{EXPERIMENTAL RESULTS}

Clinical SEP signal is recorded during scoliosis and processed by our system. Fig. 2 (a) shows a single-trial SEP response obtained after applying a stimulus. Obviously, the signal is mainly $50 \mathrm{~Hz}$ interference. Using EA, the progressively better suppression of line interference using 30 and 100 trials are shown in Fig. 2 (b) and (c) respectively. Results of eliminating the interference by ANN filter are shown in Fig. 3. From the results, we can see that having the convergence rate equal to 0.002 , training of the ANN can be completed in about $200 \mathrm{~ms}$. Afterwards, the line interference is successfully removed. Change of weights over $80 \mathrm{~s}$ interval is plotted in Fig. 4. By Eq. (6), the amplitude and phase of interference are continuously tracked with the ANN being trained on-line. From the results displayed in Fig. 5, we note that the variation of line interference strength and drifting of phase are tracked by the filter.

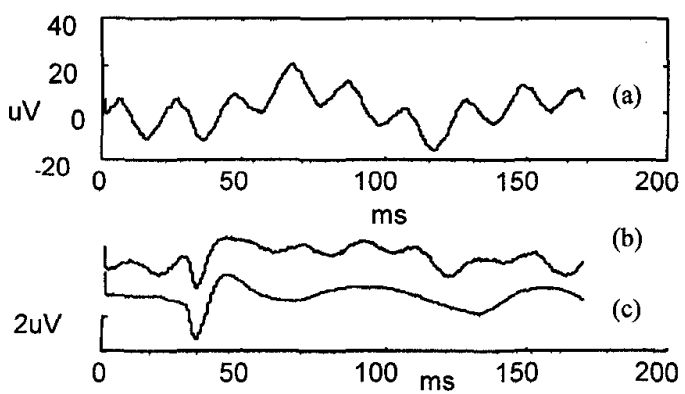

Fig. 2. SEP signal of (a) a single-trial, and results of $E A$, (b) 30 , and (c) 100 trials.

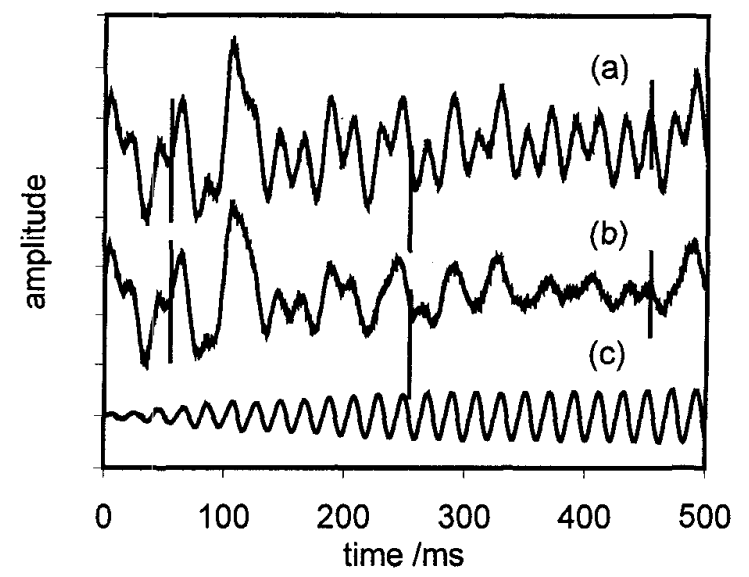

Fig. 3. Line interference cancellation by $A N N$ filter $(\mu=0.002)$ (a) observed signal, (b) filtered signal, and (c) ANN output.

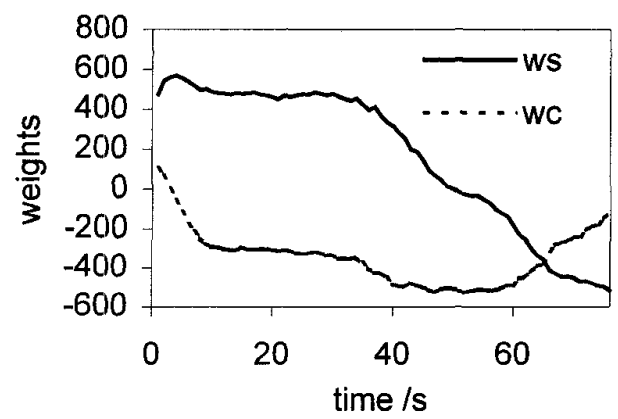

Fig. 4. Adjustment of connection weights.
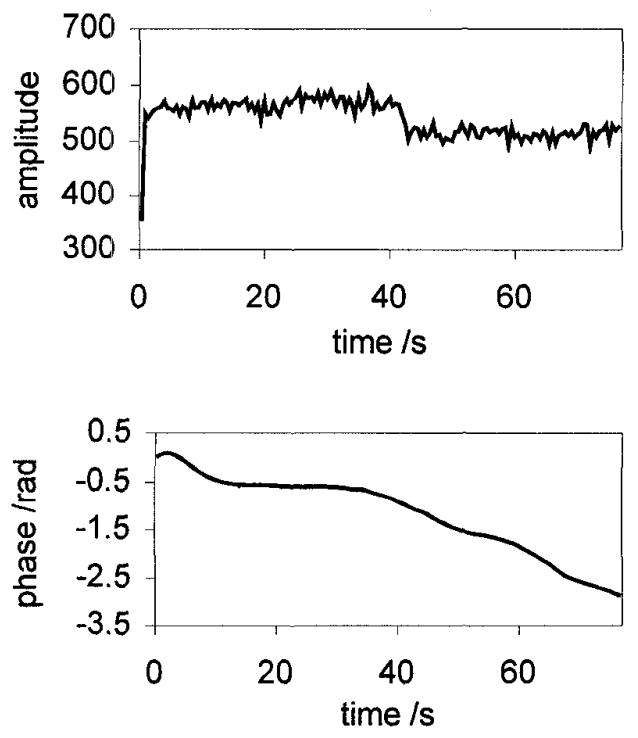

Fig. 5. Estimated amplitude and phase of sinusoidal interference. 
After canceling the line interference, the SNR of SEP signal is further enhanced by wavelet analysis. Fig. 7 (a) shows the preoperative SEP. Using this signal and the wavelet base plotted in Fig. 7 (b), the revealed SEP signal of a single-trial is displayed in Fig. 7 (d). By comparing it with the result of EA 10 trials plotted in Fig. 7 (c), our algorithm is faster to estimate the peak latency and amplitude. Thus realtime monitoring of SEP is possible.

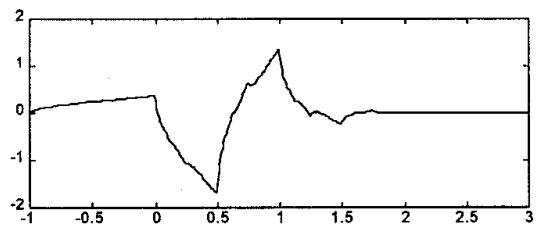

Fig. 6. Inverted Daubechies wavelet base with 4 coefficients.
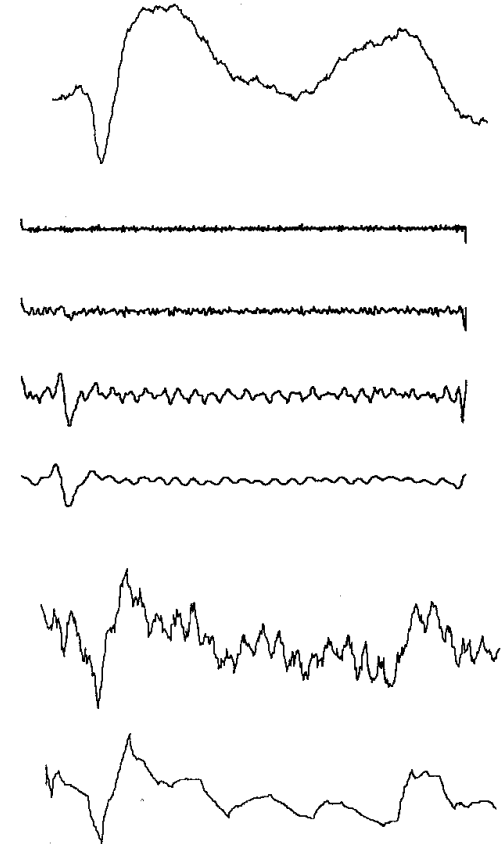

Fig. 7. Wavelet analysis (a) SEP before operation obtained by averaging 50 trials, (b) wavelet transform of (a) with 4 different scales, (c) SEP by averaging 10 trials, and (d) its SEP reconstructed from wavelet analysis with softthresholding.

\section{Discussions and Conclusions}

With the need of monitoring SEP during spinal cord operation, we are developing a fast SEP estimation system. The system is divided into pre-amplification of SEP response and advanced signal processing algorithms for measuring SEP. An ANN filter is specifically designed to eliminate the severe interference from power line. The advantages of this form of line interference filter are that it does not need an external interference signal as reference and it offers the ability of tracking the instantaneous frequency, phase and amplitude of the interference. Wavelet analysis is a sensitive algorithm to estimate change in SEP from the normal reference recorded prior to operation. Compared to traditional procedures in SEP monitoring, our system greatly improved the speed of SEP measurement and real-time monitoring of SEP becomes possible.

\section{ACKNOWLEDGEMENT}

The authors would like to thank the Research Grant Council, Hong Kong Special Administrative Region Government for financial assistance.

\section{REFERENCES}

[1] E.G. Dawson, J.E. Sherman, L.E. Kanini and M.R. Nuwer, "Spinal cord monitoring: result of the Scoliosis Research Society and the European Spinal Deformity Society survey," Spine, vol. 16, pp. 361-364, 1991.

[2] K.H. Chiappa, Evoked Potentials in Clinical Medicine. New York: Wiley, 1983.

[3] J. Schramm, et. al., Spinal cord monitoring and electrodiagnosis. Springer-Verlag, Berlin, 1991.

[4] J.C. Huhta and J.G. Webster, " $60 \mathrm{~Hz}$ interferences in electrocardiography," IEEE Trans. Biomed. Eng., vol. BME20, pp. 91-101, 1973.

[5] B. Widrow and S.D. Stearns, Adaptive Signal Processing, Englewood Cliffs, NJ: Prentice-Hall, 1985.

[6] K.S.M. Fung, W. Qiu, F.H.Y. Chan, F.K. Lam and P.W.F. Poon, "Fast adaptive evoked potential estimation using radial basis function neural network," Medical and Biological Engineering and Computing, vol. 35 Supplement, pp. 534, 1997.

[7] W.Q. Liu, M.H. Du, F.H.Y. Chan, et. al., "Real-time monitoring of VEP latency using wavelet analysis," J. South China University of Technolgy, vol. 25, no. 7, 1997.

[8] I. Daubechies, "Orthonormal bases of compactly supported wavelets," Comm. Pure Appl. Math., vol. XLI, pp. 909-996, 1988. 